\title{
DEPENDANCE OF THE METALLICITY OF PLANETARY NEBULAE WITH THE GALACTIC HEIGHT ABOVE THE DISK
}

\author{
F. CUISINIER and A. ACKER \\ Observatoire de Strasbourg, URA 1280, 11 rue de l'Université, F 67000 Strasbourg, \\ France \\ and \\ J. KÖPPEN \\ Inst.theor.Astroph., Im Neuenheimer Feld 561, D-6900 Heidelberg 1, Germany
}

\begin{abstract}
Abundances of $\mathrm{O}, \mathrm{N}, \mathrm{He}, \mathrm{S}, \mathrm{Ar}$ have been derived from the observations of $\mathrm{PN}$ at various heights above the plane with the plasma diagnosis code HOPPLA (Köppen et al. 1991). A gradient in $\mathrm{O}$ and $\mathrm{N}$ appears in the first 1000 pc. No correlation is found above.

We used the "Strasbourg-ESO catalogue of Galactic PN" (Acker et al., 1992) as a basis to select homogeneously PN above $300 \mathrm{pc}$ using Shklovskii distances calculated from the de-reddened $\mathrm{H} \beta$ fluxes. $23 \mathrm{PN}$ could be observed at the ESO $1.52 \mathrm{~m}$ telescope with a $\mathrm{B} \& \mathrm{C}$ long slit spectrograph. Abundances have been deduced via the plasma diagnosis code "HOPPLA".

The remarkable features are an absence of variation above $1000 \mathrm{pc}$ and a gradient below. The first point could be compatible with a fast collapse of the Thick Disk. The second can not be explained if we assume that metallicity is a marker of time. PN spend the most of their time near their maximum $|z|$. Thus, the galactic structures where they are originating from should be hollow, which is hard to concieve. Therefore we prefer to explain it through a variation of the age-metallicity relationship with $|z|$ for low $|z| \mathrm{PN}$.
\end{abstract}

\section{References}

Acker A., Ochsenbein F., Tylenda R., Stenholm B., Marcout J. : 1992, "The Strasbourg-ESO catalogue of Galactic Planetary Nebulae" publ. ESO,

Barker T.: 1978, ApJ 220, 193

Faundez-Abans M., Maciel W.J.: 1988, Rev.Mex.Astron.Astrof. 16, 105

Köppen J., Acker A., Stenholm B.: 1991, A\& A 248, 197

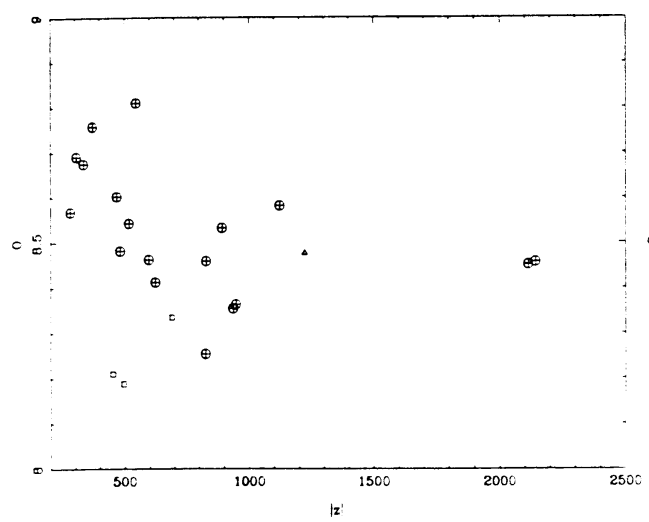

Fig. 1. oxygen abundance vs. $|z|$

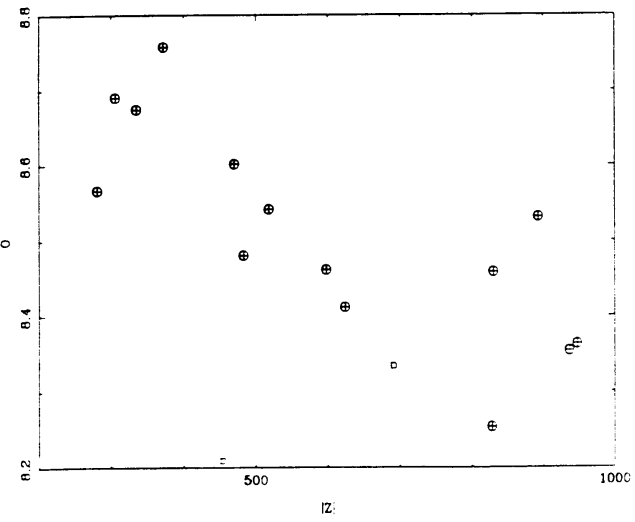

Fig. 2. lookup at the first $1000 \mathrm{pc}$ 